\title{
LDL - aferesi: trattamento extracorporeo della ipercolesterolemia
}

\author{
M. Caronna, L. Scatizzi \\ Fresenius ST, Palazzo Pignano - Cremona
}

$\mathbf{L}$

aterosclerosi rappresenta una delle principali cause di malattie coronariche e cardiovascolari, responsabili dell'elevata incidenza dell'infarto miocardico. Indagini effettuate dall'Organizzazione Mondiale della Sanità mostrano che circa il 15-20\% della popolazione americana è affetta da una forma di malattia coronarica; la stessa situazione si ritrova in Europa, tanto che le malattie cardiocircolatorie rappresentano una delle cause primarie di mortalità.

Diversi sono i fattori di rischio, più o meno elevati, che concorrono allo sviluppo precoce dell'aterosclerosi ed alla sua progressione: ipercolesterolemia, iperlipoproteinemia, ipertensione, diabete mellito, fumo di tabacco. Tuttavia il livello elevato di colesterolo nel sangue sembra essere il fattore maggiormente incriminato per lo sviluppo dell'aterosclerosi.

I lipidi plasmatici, il colesterolo, i fosfolipidi e i trigliceridi sono trasportati nel sangue sotto forma di lipoproteine. Queste sono particelle sferiche il cui co$r e$ è costituito da lipidi non polari, quali ad esempio trigliceridi ed esteri del colesterolo e si differenziano tra loro in base alla densità ed alla composizione del $\mathrm{co}^{-}$ re lipidico. La superficie delle lipoproteine è costituita da fosfolipidi, colesterolo non esterificato ed apoproteine. Anche le apoproteine presenti sulla superficie contribuiscono alla differenzia- zione delle varie classi di lipoproteine. (Tab. I)

Le LDL (Low Density Lipoprotein), le VLDL (Very Low Density Lipoprotein) e le HDL (High Density Lipoprotein) sono responsabili del trasporto del colesterolo nel sangue. Le LDL contengono una elevata quantità di colesterolo ed hanno una forte attività aterogenica, mentre le HDL mostrano spiccata attività antiaterogenica assumendo un ruolo protettivo. Le apoproteine $\mathrm{B}$ presenti nelle $\mathrm{LDL}$ sono le proteine riconosciute dai recettori specifici per le LDL, situati nella maggior parte delle cellule corporee. Il numero di recettori presenti è inversamente proporzionale al livello di colesterolemia.
Numerosi studi hanno dimostrato come un abbassamento del livello di colesterolo totale nel sangue e, in particolar modo, una riduzione di quello legato alle lipoproteine, impedisca la progressione dell'aterosclerosi e possa addirittura indurne la regressione diminuendo quindi l'incidenza di malattie cardiovascolari nonché la mortalità.

La LDL aferesi è una tecnica che impiega un sistema di circolazione extracorporeo per ridurre il livello di colesterolemia; è utilizzata principalmente per trattare pazienti affetti da "ipercolesterolemia familiare". Questa patologia autosomica dominante si presenta sia in forma omozigote che eterozigote e consiste nel-

TAB. I - CARATTERISTICHE DELLE PRINCIPALI CLASSI DI LIPOPROTEINE

\begin{tabular}{l|c|c|c|c|c}
\hline Classe & $\begin{array}{c}\text { Composizione } \\
\text { del Core }\end{array}$ & Apoproteine & $\begin{array}{c}\text { Densità } \\
\mathrm{g} / \mathrm{ml}\end{array}$ & $\begin{array}{c}\text { Diametro } \\
\AA\end{array}$ & $\begin{array}{c}\text { Mobilità } \\
\text { elettroferetica }\end{array}$ \\
\hline Chilomicroni & $\begin{array}{c}\text { Trigliceridi } \\
\text { da dieta }\end{array}$ & $\begin{array}{c}\text { AI, AII, B, } \\
\text { CI, CII, CIII }\end{array}$ & $<1.006$ & $800-5000$ & Nulla \\
\hline VLDL & $\begin{array}{c}\text { Trigliceridi } \\
\text { endogeni }\end{array}$ & $\begin{array}{c}\text { B, CI, CII, } \\
\text { CIII, E }\end{array}$ & $<1.006$ & $250-280$ & Pre-B \\
\hline LDL & $\begin{array}{c}\text { Esteri del } \\
\text { colesterolo }\end{array}$ & B & $1.019-1.063$ & $180-280$ & $B$ \\
\hline HDL & $\begin{array}{c}\text { Esteri del } \\
\text { colesterolo }\end{array}$ & AI, AII & $1.063-1.210$ & $50-120$ & $\alpha$ \\
\hline
\end{tabular}




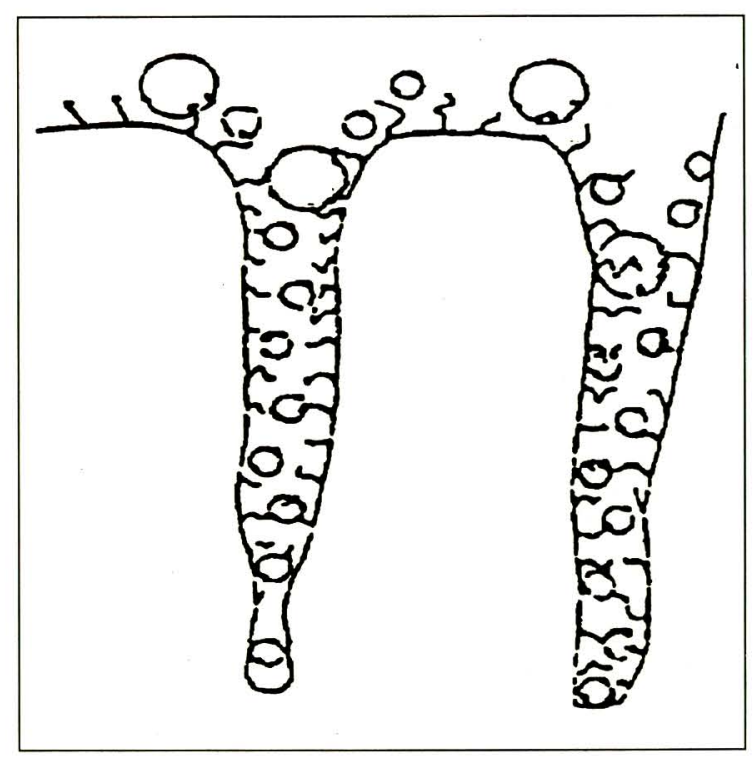

Fig. 1 - Anse delle microsfere di cellulosa (da Odaka et al, Int J Artif Organs 1986; 9: 343)

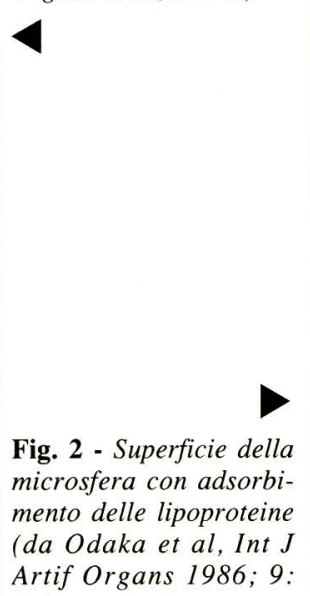
343)

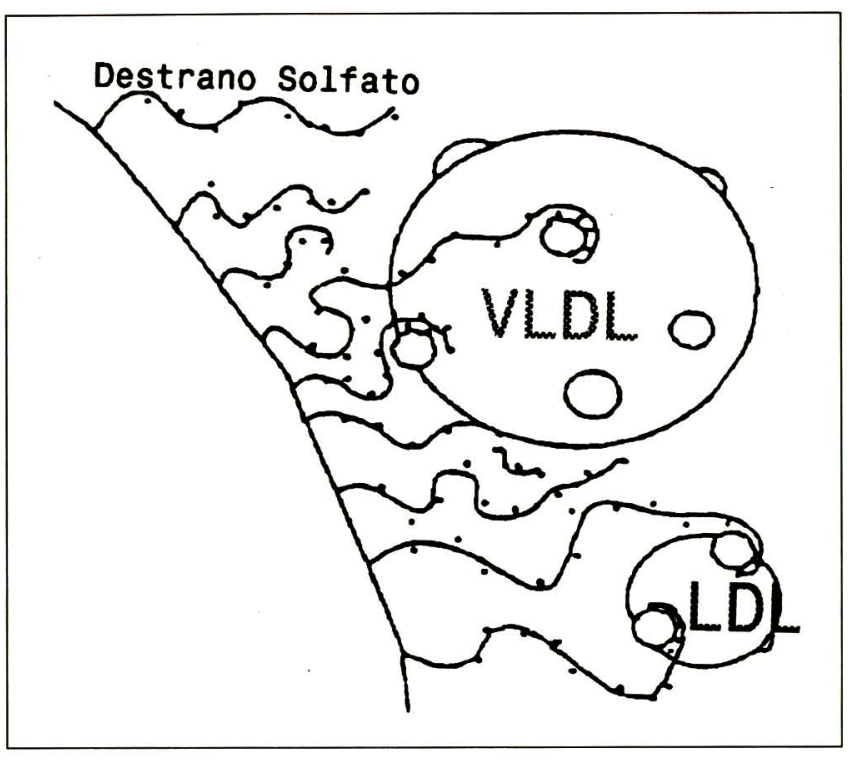

la assenza o nella riduzione dei recettori specifici per l' LDL. Nella popolazione è stata riscontrata un'incidenza di $1 / 1.000 .000$ per gli omozigoti e di $1 / 500$ per gli eterozigoti. I valori di colesterolemia in questi soggetti sono particolarmente elevati fino a raggiungere tre volte il valore normale per gli eterozigoti, e perfino cinque volte nel caso degli omozigoti.

La LDL-aferesi è indicata inoltre per soggetti affetti da "ipercolesterolemie primitive" dove non sia stata riscontrata una risposta adeguata alla terapia dietetica e farmacologica, e in presenza di ateromasia grave, per portatori di by-pass aorto coronarico e pazienti sottoposti a chirurgia vascolare maggiore.

In questi ultimi anni si sono sviluppate differenti tecniche aferetiche per la rimozione extracorporea dell' LDL colesterolo:

- plasmaferesi;

- doppia filtrazione o filtrazione a cascata;

\section{- LDL adsorbimento;}

- precipitazione extracorporea con eparina.

Per tutte le tecniche sopracitate il primo passo per poter ottenere la rimozione dell' LDL colesterolo consiste nella separazione del plasma dalla parte corpuscolata del sangue, che può avvenire tramite centrifugazione o attraverso la membra- na di un filtro specifico per la plasmaseparazione. Quest'ultima tecnica permette l'estrazione di plasma con un basso contenuto piastrinico riducendo la possibilità di intasamento delle colonne adsorbenti.

Il Liposorber LA-15 System proposto da Kaneka fu approvato in Giappone nel 1987 per il trattamento di severe forme di ipercolesterolemia familiare. Si tratta di un sistema altamente selettivo che rimuove le LDL, le VLDL e le Lp(a) dal plasma mediante adsorbimento su colonne. Ogni colonna contiene $150 \mathrm{ml}$ di destrano solfato, fissato chimicamente con legami covalenti a delle microsfere di cellulosa sino a costituire un gel. Lo sviluppo superficiale di tutte le microsfere è paragonabile alla superficie di un campo di calcio.
L'adsorbimento dell'LDL sul destrano solfato è ottenuto tramite l'interazione elettrostatica tra le Apo lipoproteine B di carica positiva e le catene di destrano solfato cariche negativamente. Questa interazione ha una specificità paragonabile a quella biologica tra LDL e i recettori cellulari.

La particolare struttura delle microsfere che presentano delle strette anse in cui le lipoproteine più grandi non possono legarsi al destrano solfato, causa un minore adsorbimento delle VLDL rispetto alle LDL.

Le HDL invece non presentano apoproteine B di carica positiva e quindi non si adsorbono al destrano solfato mantenendo invariata la concentrazione prima e dopo il trattamento (Figg. 1 e 2).

Le lipoproteine LDL e VLDL si possono successivamente staccare dal destrano
Fig. 3 - Capacità costante di adsorbimento dell' $L D L$ colesterolo dopo ripetute rigenerazioni delle colonne di destrano solfato.

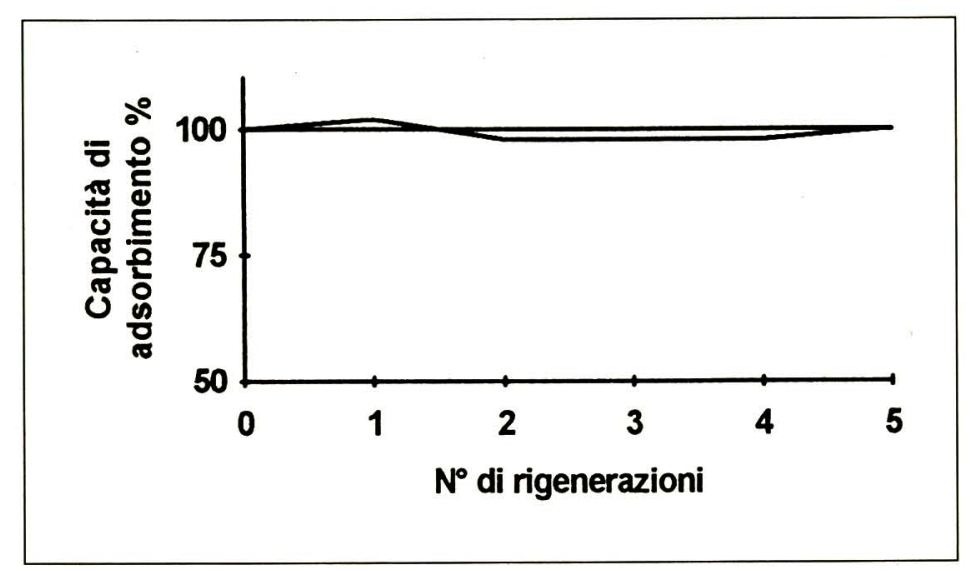


utilizzando una soluzione di $\mathrm{NaCl} 0.7 \mathrm{M}$ ottenendo così una completa rigenerazione della colonna.

In questo modo ogni colonna è in grado di mantenere alta la capacità di adsorbimento che nel corso della seduta rimane intorno al $100 \%$ (Fig. 3).

Poiché l'adsorbimento è estremamente selettivo e specifico, la concentrazione delle altre componenti plasmatiche quali l'albumina, la globulina, l'HDL, le proteine totali rimane inalterata in corso di LDL aferesi o viene alterata in maniera del tutto marginale (Fig. 4).

Per effettuare la LDL-aferesi mediante adsorbimento su colonne di destrano solfato, la Kaneka ha realizzato l'apparecchiatura MA-01. Questa utilizza un microprocessore per regolare e controllare tutte le fasi del trattamento, il prelavaggio e il priming del circuito extracorporeo, il processo di separazione del plasma dal sangue, l'adsorbimento delle LDL e la rigenerazione delle colonne. Al termine del trattamento, quando è stato raggiunto il volume di plasma da trattare, subentra una fase automatica di reinfusione del sangue e del plasma.

Come anticoagulante viene utilizzata l'eparina che viene infusa tramite una pompa a siringa integrata nella macchina.

Per la separazione del plasma si utilizza un filtro a fibre cave in polisulfone, di solito viene trattato fino ad 1.5 volte il volume totale di plasma del paziente $(70$ $\mathrm{mg} / \mathrm{kg}$ ). Due colonne contenenti ciascuna $150 \mathrm{ml}$ di destrano solfato sono utilizzate per adsorbire l' LDL. Dopo il passaggio di un volume di $500 \mathrm{ml} \mathrm{di} \mathrm{pla-}$ sma, la colonna di destrano solfato è satura di LDL e automaticamente la macchina devia il plasma sull'altra colonna. Il plasma contenuto nella prima colonna (1) viene reinfuso al paziente perfondendo $140 \mathrm{ml}$ di soluzione fisiologica nella colonna. A questo punto il plasma contenuto nella seconda colonna (2), che nel frattempo si era riempita di plasma, si collega alla linea di ritorno venosa, e la soluzione fisiologica della prima colonna (1) viene rilasciata allo scarico. Successivamente si infondono $105 \mathrm{ml} \mathrm{di}$ soluzione di rigenerazione nella colonna (1) per rimuovere le LDL dal destrano solfato. Il ciclo di rigenerazione ha termine con il lavaggio con il passaggio di $355 \mathrm{ml}$ di fisiologica isotonica nella colonna (1).

Per evitare l'infusione al paziente di soluzione ipertonica di rigenerazione, sulla

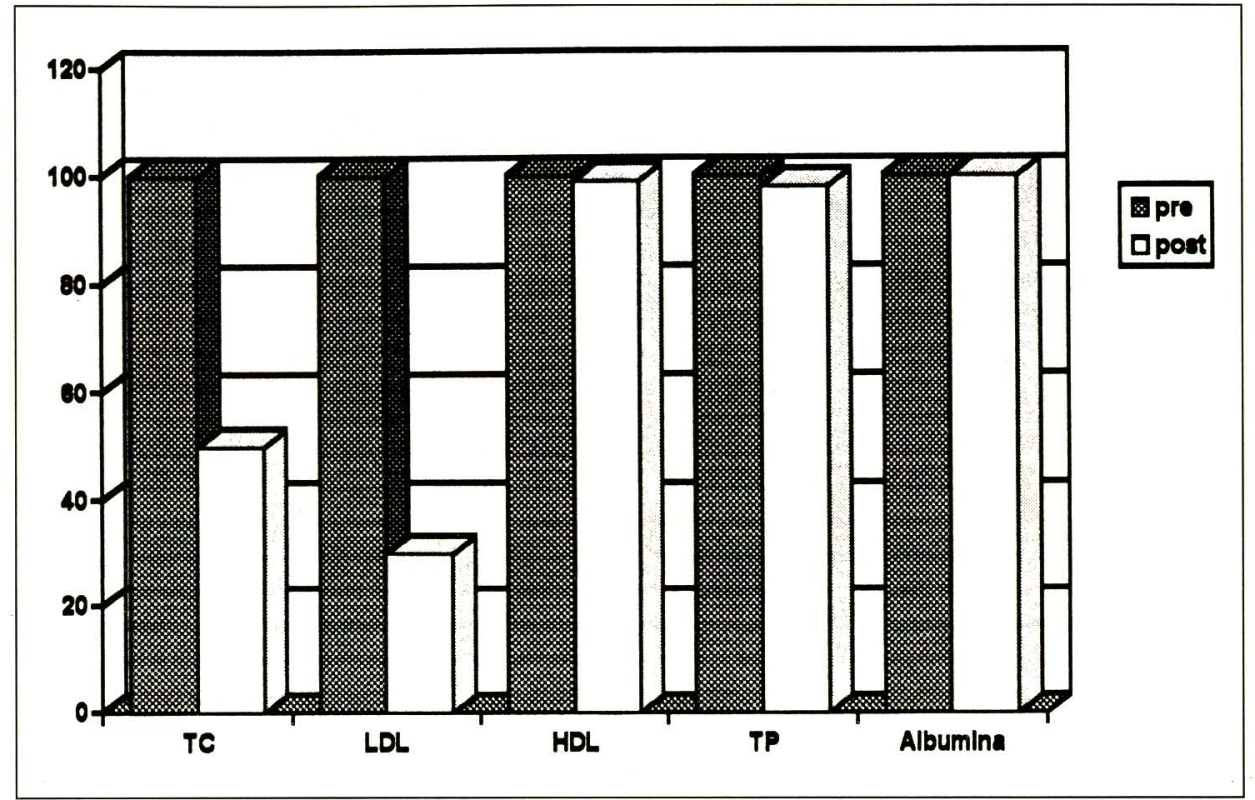

Fig. 4 - Variazioni dei livelli delle componenti plasmatiche pre e post aferesi.

\section{Descrizione del Sistema Kaneka}

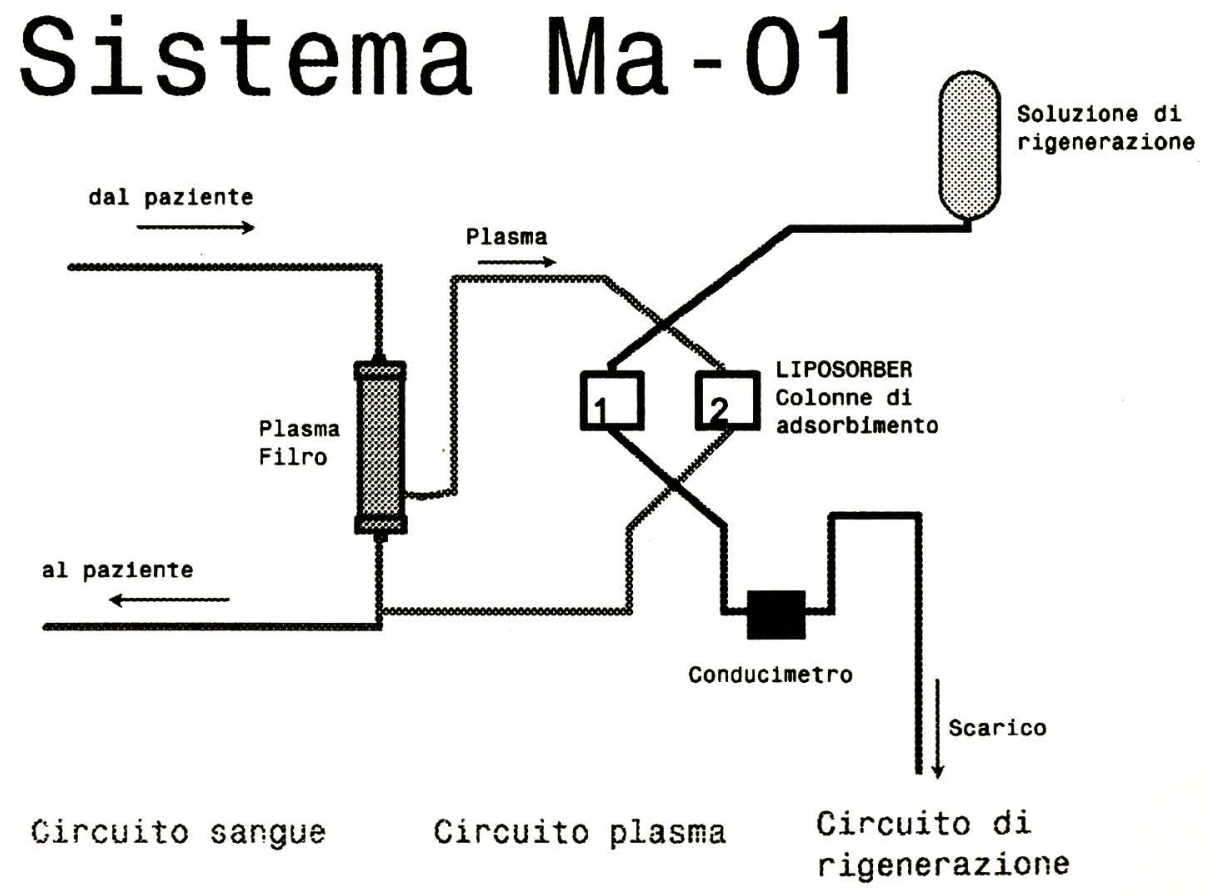

linea di scarico è inserito un conducimetro che verifica l'isotonicità della soluzione di lavaggio. Il ciclo di rigenerazione si ripete alternativamente sulle due colonne dopo $600 \mathrm{ml}$ di plasma, eccetto per la prima rigenerazione che avviene dopo $500 \mathrm{ml}$ poiché la prima quota di plasma estratta è più ricca di LDL. Il plasma dopo esser passato attraverso la colonna adsorbente si miscela con la parte corpuscolata del sangue proveniente dal plasma separatore. Il sangue così miscelato viene riportato alla temperatura di $37^{\circ} \mathrm{C}$ e reinfuso al paziente. Il circuito 
extracorporeo ha un volume totale di 400 $\mathrm{ml}$. Le colonne sono monouso e la durata del trattamento varia da 2.5 a 3 ore in funzione del volume di plasma da trattare e del flusso di sangue ottenibile.

Il pannello frontale dell'apparecchiatura è provvisto di un display digitale che visualizza i valori del flusso sangue e plasma, il volume di plasma trattato e le pressioni nei vari punti del circuito extracorporeo.

La pompa sangue si regola in base al valore della pressione di aspirazione del sangue della vena diminuendo automaticamente quando la pressione diventa troppo negativa. Dopo ogni arresto della pompa sangue la velocità della pompa ritorna al valore desiderato con un incremento di $(20 \mathrm{ml} / \mathrm{min}) / \mathrm{min}$. Di solito i pazienti sottoposti a questo trattamento non dispongono un accesso vascolare efficiente come la fistola a-v, quindi l'avvio della pompa sangue a "rampa" serve per ridurre il collabimento del vaso durante l'aspirazione del sangue.

Il sistema di circolazione extracorpoea comprende:

- una pompa sangue, una plasma ed una per la rigenerazione;

- una pompa eparina;

- trasduttori di pressione;

- rilevatori bolle d'aria e di perdite ematiche;

- rilevatori di mancanza di fluido;

- rilevatori di livello;

- un conducimetro;

- un riscaldatore sangue a piastre;

- clamp automatiche.

Il sistema di rigenerazione delle colonne permette di trattare fino a 10 litri di plasma per seduta con una capacità di adsorbimento invariata. Non sono state evidenziate particolari controindicazioni nell'utilizzo del sistema se non quelle proprie dei trattamenti extracorporei.

È indicata la sospensione degli Ace-inibitori e di farmaci ipotensivi prima della seduta aferetica in quanto possono indurre gravi eventi ipotensivi durante il trattamento.

Obiettivo principale della LDL-aferesi è quello di ridurre drasticamente la concentrazione plasmatica del colesterolo legato alle lipoproteine al fine di raggiungere un livello di colesterolemia non potenzialmente aterogenico e di riuscire inoltre a mantenere più basso possibile il livello medio di colesterolo.

Il Liposorber LA-15 System (ora commercializzato in Italia da Fresenius ST) permette di ottenere una riduzione pari al 60-70\% della concentrazione di colesterolo preaferesi ed ha una elevata specificità per le LDL. Poiché dopo la seduta di aferesi si verifica un rebound della concentrazione di colesterolo con ampia variabilità sia intra che interindividuale, il trattamento va ripetuto con una frequenza personalizzata che varia dai 7 ai 15 giorni circa. Inoltre per valutare la frequenza e la continuità del trattamento devono essere presi in considerazione sia i risultati degli esami di laboratorio sia le risposte ottenute dalla terapia dietetica e farmacologica associata.

Il sistema Kaneka, altamente selettivo e specifico per la rimozione dell'LDL colesterolo, è in grado di ridurre sensibilmente anche la concentrazione delle $\mathrm{Lp}(\mathrm{a})$. Queste glicoproteine legate alle ApoB, considerate fattori di rischio indipendenti, sembrano avere un ruolo rilevante nella malattie cardiovascolari.

I benefici ottenuti dai pazienti sottoposti a questa terapia possono quindi essere riassunti in una efficace riduzione e scomparsa della maggior parte dei depositi periferici di colesterolo (xantomi), nella regressione delle lesioni ateromatose dei vasi ed in un conseguente e significativo aumento della sopravvivenza.

\section{BIBLIOGRAFIA}

1. Kitano Y, Thompson GR. Role of LDL apheresis in the management of hypercholesterolaemia. Transfus Sci 1993; 14: 3.

2. Gordon BR, Saal SD, Kelsey $\mathrm{SF}$. The treatment of refractory familial hypercholesterolaemia using the liposorber LA-15 system. In: Gordon BR, Gotto AM eds. The treatment of severe hypercholesterolemia: can we impact disease course? Proceedings of a Symposium November 9, 1991 Anaheim, California. Princeton, NJ: Excerpta Medica: Inc.

3. Olbricht CJ, Schaumann D, Fischer D. Anaphilactoid reactions, LDL-apheresis with dextran sulfate and ACE-inhibitors. Lancet 1992; 340: 908-9.

4. Kark JD, Sandholzer C, Friedlander Y, Utermann G. Plasma $\mathrm{Lp}(\mathrm{a})$, apolipoprotein(a) isoforms and acute myocardial infarction in men and women: a case control study in the Jerusalem population. Atherosclerosis 1993; 98: 139-51.

5. Yamamoto K, Nakashima Y, et al. Effect of low-density lipoprotein apheresis on reduction of lipoprotein(a) in patients with familial hypercholesterolemia: a multicenter study. Nutrition, Metabolism and Cardiovascular Diseases 1993; 3 : 23-7.

6. Odaka M, Kobayashi H, Soeda $\mathrm{K}$, et al. Adsorption of lipoprotein containing apolipoprotein-B through plasma separation for treatment of familial hypercholesterolemia. Int $\mathrm{J}$ Artif Organs 1986; 9:343-8. 